Nomenclature

$\begin{array}{llr}D & =\text { cumulative undersize percent } & {[\%]} \\ d_{f} & =\text { particle size of feed material } & {[\mu \mathrm{m}]} \\ d_{p} & =\text { particle size of product } & {[\mu \mathrm{m}]} \\ H d & =\text { Hardgrove grindability } & {[-]} \\ M_{B} & =\text { weight of grinding medium in mill } & {[\mathrm{g}]} \\ \Delta S & =\text { specific surface area produced } & {\left[\mathrm{cm}^{2} / \mathrm{g}\right]} \\ T & =\text { residence time } & {[\mathrm{s}]} \\ W_{f} & =\text { weight rate of particles fed } & {[\mathrm{g} / \mathrm{min}]} \\ W_{t} & =\text { total mass flow rate of air through both } & \\ & & \text { grinding and injector nozzles } \\ & & {[\mathrm{g} / \mathrm{min}]} \\ \rho_{p} & =\text { density of feed material } & {\left[\mathrm{g} / \mathrm{cm}^{3}\right]}\end{array}$

\section{Literature Cited}

1) Dotson, J. M.: Ind. Eng. Chem., 54, 62 (1962).

2) Honma, T., M. Hasegawa and Y. Asaba: Kagaku Kogaku Ronbunshu, 6, 527 (1980).

3) Kürten, H. and H. Rumpf: Chem.-Ing.-Tech., 38, 331 (1966).

4) Ramanujam, M. and D. Venkateswarlu: Powder Tech., 3, $92(1969 / 70)$.

(Presented at the Spring Meeting of the Mining and MetalIurgical Institute of Japan, at Tokyo, April, 1980.)

\title{
EFFECT OF SCRAPING ANGLE, OR THICKNESS OR TIP ANGLE OF BLADES ON POWER CONSUMPTION OF A FLUID-LOADED HINGED SCRAPER BLADE
}

\author{
MICHIHARU TOH AND YASUHIRO MURAKAMI \\ Department of Industrial Chemistry, Kurume National Technical College, Kurume 830
}

In the previous paper ${ }^{3)}$, the power consumption of the fluid-loaded hinged scraper blade was measured when the scraping angle, or the number or width of the blades, was changed. Results were compared with prediction by the theoretical equation of power consumption of the blade and agreement was fairly good.

In this paper, the effect of the scraping angle, or the thickness or tip angle of the blades, on the power consumption of the blade was studied in detail and the experimental results were compared with the above theoretical equation.

\section{Experimental}

The experimental apparatus and procedure used were almost the same as described in the previous papers $^{1,2)}$. A brass scraper blade with a length of $0.11 \mathrm{~m}$ and a width of $0.028 \mathrm{~m}$ and a syrup solution having viscosity range of $10.8-32.9 \mathrm{~Pa} \cdot \mathrm{s}$ and density range of $1390-1410 \mathrm{~kg} / \mathrm{m}^{3}$ were used. Experiments were performed in which the scraping angle $\alpha$, the thickness $a$ and the tip angle $\eta$ of the blades were changed as follows: $\alpha=\pi / 9, \pi / 6,2 \pi / 9, \pi / 4,5 \pi / 18, \pi / 3$; $a=1,2,3,5 \mathrm{~mm} ; \eta=\pi / 6, \pi / 3, \pi / 2$.

To observe the effects of these factors in detail,

Received October 9, 1981. Correspondence concerning this article should be addressed to M. Toh. Y. Murakami is at Dept. of Chem. Eng., Kyushu Univ., Fukuoka 812 only one blade was used. Thus the bottom and the lid plate of the vessel were equipped with the bearings shown in Fig. 1 to prevent shaft deflection and to adjust the center of the vessel precisely to that of the shaft. Before measurement of power consumption, the scraping angle $\alpha$ was measured by the apparatus with a protractor shown in Fig. 2 and the degree of contact between the edge of the blade and the vessel wall was checked by the light of the miniature lamps shown in Fig. 3. That is, the inserted lamps in front of the blade about the scraped point were moved vertically and the blade was set in a state where leakage of light from the lamps at the back of the blade was as little as possible. During the experiment, the electrical resistance between the edge of the blade and the vessel wall was measured in the same way as described in the previous paper to confirm the degree of contact. The value of the resistance was within $10 \Omega$ in these experiments, in comparison with values varying from 0 to $10 \mathrm{k} \Omega$ in the previous experiments ${ }^{2,31}$.

\section{Experimental Results}

Experimental results are compared with the theoretical equation in the previous paper ${ }^{3}$ shown in Figs. $\mathbf{4 , 5}$ and 6 . In these experiments, the degree of contact between the edge of the blade and the vessel wall 


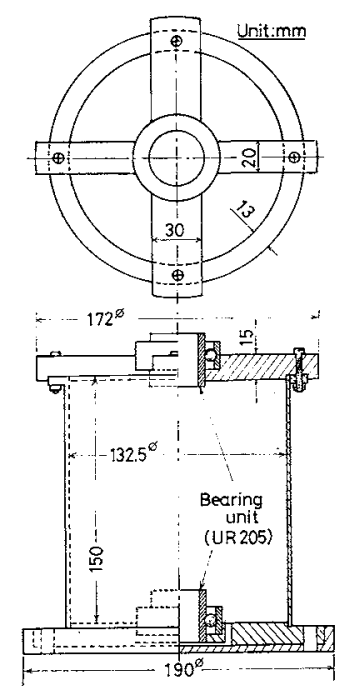

Fig. 1 Dimensions of vessel equipped with bearings

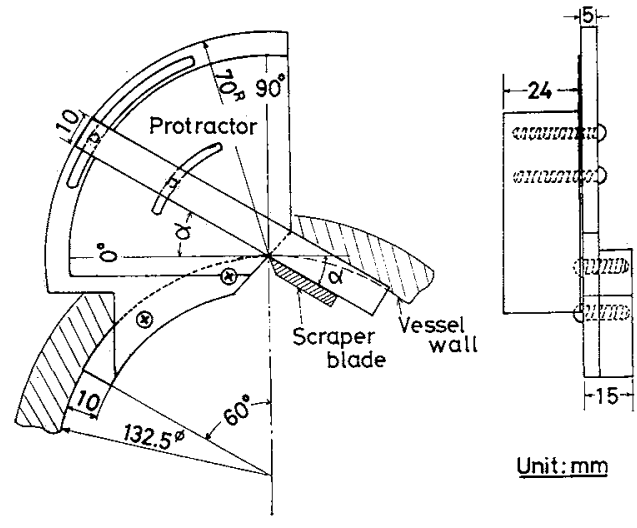

Fig. 2 Illustration of apparatus with protractor

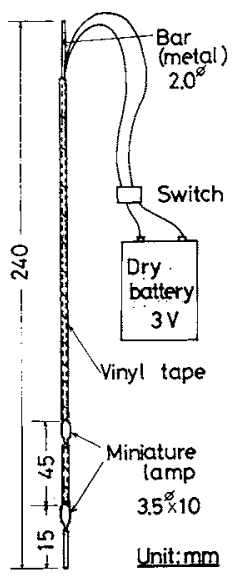

Fig. 3 Illustration of apparatus with miniature lamps

was confirmed by the light of the miniature lamps and by confirming that the elctrical resistance was very small. We could thus consider the value for the kinematic friction coefficient $f$ to be 0.15 instead of 0.10 , the value in the previous papers ${ }^{2,3}$. Figure 4

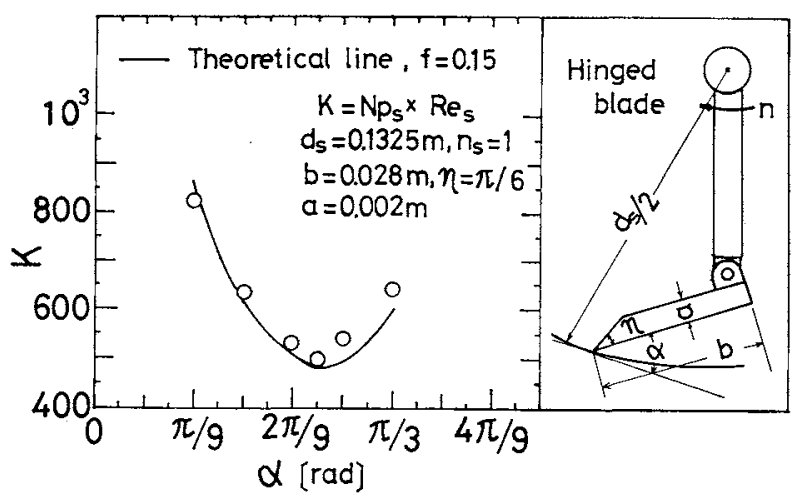

Fig. 4 Comparison between experimental data and theoretical values for different scraping angles

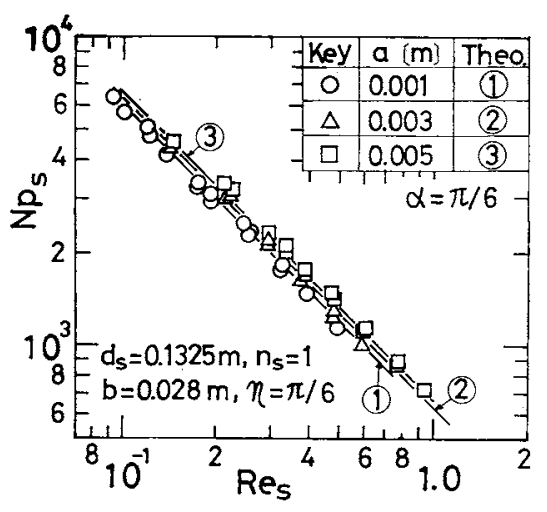

Fig. 5 Comparison between experimental data and theoretical values for different thicknesses

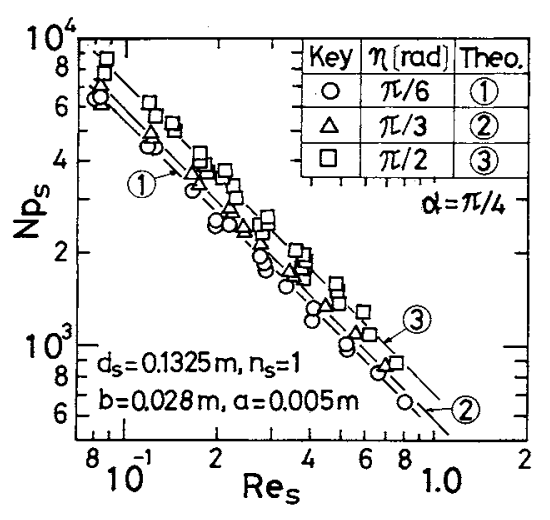

Fig. 6 Comparison between experimental data and theoretical values for different tip angles

shows the relationship between the coefficient of correlation equation of power consumption $K$ and the scraping angle $\alpha$ for $\eta=\pi / 6$ and $a=2 \mathrm{~mm}$. The tendency of experimental results between $K$ and $\alpha$ agreed fairly well with the theoretical value and the power consumption at $\alpha=\pi / 4$ was the minimum. Figure 5 shows the relationship between Power number $N p_{s}$ and Reynolds number $R e_{s}$ of the scraper blade in experiments using blades of different thickness for $\eta=\pi / 6$ and $\alpha=\pi / 6$. Experimental results agreed 
fairly well with the theoretical value and the power consumption increased very little as the thickness of the blade increased from $1 \mathrm{~mm}$ to $5 \mathrm{~mm}$. Figure 6 shows the relationship between $N p_{s}$ and $R e_{s}$ in experiments using blades of different tip angle for $\alpha=$ $\pi / 4$ and $a=5 \mathrm{~mm}$. Experimental results pretty well agreed with the theoretical value and the power consumption increased considerably as the tip angle of the blade increased from $\pi / 6$ to $\pi / 2$.

\section{Conclusion}

It was found that the scraping angle, and the thickness and tip angle of the blade considerably influenced the power consumption of the blade. The experimental results agreed fairly well with the theoretical equation in the previous paper $^{33}$.

\section{Acknowledgments}

We wish to thank Messrs. T. Gondoh, H. Oyama, T. Kitahara and $T$. Suetake for their help in carrying out the experiments. This work was supported in part by the Science Research Foundation of the Ministry of Education, Science and Culture, Japan, Grant No. 565314.

\section{Nomenclature}

$b$

$d_{s} \quad=$ diameter of scraper blade (=I.D. of vessel)

$N p_{s} \quad=$ power number of scraper blade $P_{s} / \rho n^{3} d_{s}^{5}$

$n \quad=$ rotational speed of scraper blade

$n_{\mathrm{s}} \quad=$ number of scraper blades $\quad[-]$

$P_{s} \quad=$ power of scraper blade [W]

$\operatorname{Re}_{s} \quad=$ Reynolds number of scraper blade $d_{s}^{2} n \rho / \mu$

$\alpha \quad=$ scraping angle (back) $\quad$ [rad]

$\eta \quad=$ tip angle of scraper blade $\quad$ [rad]

$\mu \quad=$ viscosity of syrup solusion $\quad[\mathrm{Pa} \cdot \mathrm{s}]$

$\rho \quad=$ density of syrup solution $\quad\left[\mathrm{kg} / \mathrm{m}^{3}\right]$

\section{Literature Cited}

1) Toh, M. and Y. Murakami: Kagaku Kogaku Ronbunshu, 6, 417 (1980).

2) idem: ibid., 6, 423 (1980).

3) idem: ibid., 7, 90 (1981).

(Presented at the 46th Annual Meeting of The Soc. of Chem. Engrs., Japan, at Nagoya, April 1981.)

\section{ABSTRACTS OF KAGAKU KOGAKU RONBUNSHU VOL. 8, NO. 3, MAY, 1982}

Continuous Measurement of Local Concentration by an Optical Fiber Probe, Hiroyuki Hatano and Masaru Ishida (Tokyo Inst. of Tech.)

Kagaku Kogaku Ronbunshu, 8, 219-224 (1982)

A method for measuring continuously the local concentration in three-dimensional equipment is proposed. This method, in which a fine optical fiber probe was used, is based on the absorptiometry technique.

The measurement of concentrations of blue ink in water, $\mathrm{NO}_{2}$ gas in air, and fine kaolin powder suspended in water showed its high applicability.

It was also used to measure the tracer gas concentration in a gas-solid fluidized bed. It was found that the tracer concentration was continuously measured not only in the bubble phase but also in the emulsion phase with high-speed response.

Carryover from a Liquid Pool with Vertical Air Flow, Tadashi Iguchi (Japan Atomic Energy Res. Inst.)

Kagaku Kogaku Ronbunshu, 8, 225-231 (1982)

An experiment was performed to investigate the carryover flow rate from a liquid pool above an orifice plate through which a vertical air flow was maintained. Based upon visual observations, the major causes of the carryover phenomena were classified into three types: (1) entrainment generated by the breaking of a bubble at the surface of the liquid pool; (2) entrainment generated by the disintegration of a large-amplitude wave or bridge near the water surface; and (3) carryover by vertical oscillations of the water surface. It was found that the carryover rate is governed mainly by phenomena near the surface and partially by the geometry of the inlet orifice. To predict the type (2) carryover rate, a non-dimensional correlation was derived on the basis of a momentum exchange assumption.

Pressure Profile of Bingham Fluids in Screw Extruders of Constant Pitch-Straight Taper Type and Varying Pitch Type, Mompei Shirato, Toshiro Murase and Masashi Iwata (Nagoya Univ.)

Kagaku Kogaku Ronbunshu, 8, 232-239 (1982)

Simplified methods for analysing the pressure profile of Bingham fluids in screw extruders are presented. The conventional flow equation for Bingham fluids is modified in definition of an average differential viscosity. Simplified equations for predicting the pressure distribution in extruders of both constant pitch-straight taper type and constant channel depth-varying pitch type are derived on the flat-plates representations of the screw channel, in consideration of the fact that the leakage comprises both pressure flow and drag flow components at right angles to the helix. Graphical evaluation of a correction 